\title{
Strategic and Tactical Hospital Bed Management in Belgium and in France
}

\author{
D. Gossart ${ }^{1}$, N. Meskens ${ }^{1, *}$, and A. Guinet ${ }^{2}$ \\ ${ }^{1}$ UcLouvain - Louvain School of Management - Campus Mons \\ Management department \\ Chaussée de Binche 151 - 7000 Mons - Belgium \\ Tel.: +3265323350 \\ gossartdafucam.ac.be, meskens@fucam.ac.be \\ ${ }^{2}$ INSA - Lyon, France \\ alain.guinetainsa-lyon.fr
}

\begin{abstract}
Nowadays, lots of healthcare establishments decide to integrate a hospital bed management department, while taking into account the financial impact on the budget, the disruptions and factors influencing the bed planning as e.g. the type of care units management and the existing networks between hospitals and satellite structures. Lots of constraints will also have an impact while optimizing the available resources: accepting the emergency case and therefore delaying the planned patient, adding additional beds within a care unit and studying at the same time the collateral costs, opening or closing beds related to a specialty, etc.
\end{abstract}

Keywords: hospital bed management, strategic and tactical management, collaborative networks, mono site hospital, multi-site hospital.

\section{Introduction}

Nowadays, the hospital management takes place in an increasingly competitive environment and it is therefore essential to focus on delivering high quality care to patients. Lots of healthcare establishments have decided to center the organization on the hospital bed management while stimulating changes and systems improvements.

The aim of the hospital bed management is to allocate beds to elective and nonelective patients while taking into account several constraints. This task is very complex. These difficulties come from on one hand, the uncertainty related to the length of stay, the arrival of emergencies and on the other hand from an important number of constraints: single-sex rooms, the nursing staff competencies, the notion of asepsis, the social or medical isolations and of course the patient's preferences in terms of room type. Moreover, the type of care units organization and also the existence and the form of the collaborative networks can have an impact on bed

\footnotetext{
* This research is financed by the Interuniversity Attraction Poles Program - Belgian State Belgian Science Policy.
} 
management. In the current economic context, collaborative networks appear to be a good compromise to reduce costs and to improve healthcare delivery. Hospital bed management has a crucial impact on the hospital financing and on the care units occupancy rates all along the patient's pathway.

This research will focus on the strategic and tactical levels due to the fact that it is crucial to achieve the long-term objectives, while developing plans and allocating as best as possible the existing resources. A strategic type of management consists in determining on the long run a number of rooms and a bed capacity in function of one or more future evolution axis of the healthcare establishment. From a tactical point of view, it focuses on pre-planning admissions, on forecasting demand, on human and material resources needed in function of seasonal effect, while measuring the impact that emergencies would have on this planning.

This paper will be structured into five parts: after the introduction, the hospital bed management will be tackled as well as the factors influencing bed management with a special focus on the influence of the collaborative networks. The third part will then expose the encountered issues linked to bed management within the framework of the visited hospitals. The fourth section will go back over the reformulation proposition of the problems. Finally, the conclusion will then sum up few avenues that undoubtedly have to be explored and courses of improving that could be realized in further stages of this research.

\section{Hospital Bed Management}

As an optimal bed management is nowadays becoming crucial for hospitals, it is important to highlight factors influencing the bed management. To realize this, we used two methods. On one hand, we made a short literature review and on the other hand, we visited eighteen Belgian and French hospitals whereas people occupying different functions were interviewed. An overview of these visits is presented in the following section.

Different compulsory factors complicate and impact on the bed management e.g. uncertainty related to the length of stay and uncertainty related to the number of emergency cases. Other elements also need to be taken into account in order to build up beds planning: single-sex rooms, the importance of putting a pathology in the appropriate care unit, the notion of asepsis, the social or medical isolations, the patient's preferences in terms of room type, etc. These are key elements that bed managers have to keep in mind. In the literature, some authors approached these elements.

In a strategic point of view, Nguyen et al. [1] used an average duration of stay to determine the necessary bed capacity. In a tactical point of view, Cochran and Roche [2] used a methodology also based on queuing network in order to improve the capacity of emergency service according to patients' type, the random entrances, etc. Wang [3] studied the bed resources allocation taking into account hospitalization demands, bed capacity and incomes coming from the activity, while optimizing the incomes and minimizing of additional beds. In an operational point of view, Vassilacopoulos [4] used a simulation model in order to improve the bed utilization while taking into account specific operational constraints (level of emergency, bed occupancy and length of waiting list). Furthermore, Walczak et al. [5] used the neural 
network theory to estimate the duration of stay of each patient coming from the emergency room. This developed resource permits to physicians and staff people allocating beds in function of estimated length of stay.

Moreover, two main factors influence the hospital bed management: the type of care units organization, explained hereunder and the collaborations existing between hospitals and their environment.

Three main ways of care units management need to be distinguished (Ben Bachouch et al., [6]): management by specialty (coded as $\mathrm{S}$ in Tables 1 and 2), management by length of stay (coded as LOS) and management by groups of specialties (coded as GS).

The other important factor in terms of hospital bed management is linked to the different networks that mainly exist between hospitals (i.e. multi-site architecture or not) and between a hospital and satellite structures as rest homes or rehabilitation centres. A collaborative network is expressed as links between several organisations, whereas members have a key role, in order to achieve and solve goals. Axelrod [7] said that "trust and reciprocity are the shadow of the future: the more trust and reciprocity in the network, the greater the ability of the network to accomplish shared goals." According to Milward and Provan [8], four types of management network exist: service implementation network based on joint production of services, information diffusion network sharing information across organizations, problem solving network and community capital network building social relationships and capital across members. The important element to take into account is the way of evolving and thus the created dynamic around the network. According to the majority of bed managers we met, it is essential to develop new competencies, to enhance cohesion between all members and to induce over and over again new connexions and exchanges. Data integration is therefore a crucial element due to the importance of having a general view on functioning. Indeed, collaborations within the healthcare environment mainly permit collecting data about patients and exchanging clinical data.

\section{Overview of Visited Healthcare Establishments}

In order to better analyze the current situation in Belgium and in France, fourteen Belgian hospitals and four French hospitals have been visited from end 2009 to beginning 2010. The aim was to diversify as much as possible the visits in order to get a picture of the different hospitals in terms of size (number of beds), organization (multi or mono site) and type (university hospital or not). The aims of these visits were to clearly understand how the hospital beds are managed and what kind of issues they encounter every single day.

Tables 1 and 2 below indicate the principal characteristics of visited hospitals, respectively regarding mono site and multi-site hospitals. Healthcare establishments can be categorized according to their size: "Big" means that the size is superior to 500 beds, "Average" stands for a structure containing between 200 to 500 beds while hospitals with less than 200 beds are characterized as "Small". Hospitals can also be categorized as multi-site or mono site (i.e. built up on one site or laid out in different sites). The fourth column presents the different types of hospitals. Three types of hospitals mainly exist in Belgium: "G" stands for general hospitals, "U" for university hospitals and "GCU" for general hospital with university characters. In France, the main types are: "CH" stands for hospital centres, "CHU" for university hospitals and 
"CHR" for regional hospital centres. Finally, the fifth column goes back over the type(s) of management existing in the care units.

In order to better visualize the daily problems encountered by each visited hospitals, Tables 1 and 2 below sum up the following issues:

a) if the patient is not in the appropriate care unit related to his pathology, it implies an organizational problem regarding the physicians' visits, a problem related to the nursing staff competencies, not always used to care specific pathologies and also problems regarding his internal transfer that can be costly, especially in terms of stretch-bearing activities, administration, etc.

b) it is not always possible to give the type of room that the patient wanted (especially private rooms). Indeed, the bed allocation needs to take into account different elements: length of stay that must be estimated and that are not always known before the beginning of the hospitalization, the patient's age, single-sex rooms, the nursing staff competencies, the notion of asepsis and isolation and of course the patient's preferences in terms of room type. It is thus not always easy to give a private room when other elements need to be taken into account uppermost. Indeed, some visited hospitals consider this patient's preference as the most/least important element and will prioritize or not this criteria in comparison with other factors.

c) patients sometimes stay more than twenty-four hours in the temporary emergency service. Indeed, lots of hospitals must welcome a huge number of emergencies, disrupting the original planning. Generally, hospitals have a temporary emergency service, coupled with the emergency department, that allows welcoming patients for maximum twenty-four hours when no other beds are available in the appropriate medical service. Nevertheless, it happens that patients stay for longer than one day due to the lack of beds in the care units, consequently implying an obstruction in the process of hospitalization.

d) it happens to transfer externally patients to other hospitals. Therefore, it implies a loss of patients. In the worst case, if no bed (even additional bed added for a shortterm period) can be found inside the hospital or in another site belonging to the same structure, an external transfer must be planned, even if this transfer means a possible escape of patients in favor of other establishments. Indeed, it goes further than a loss of one hospitalization: it also means a loss of one or more consultations after the hospitalization and even a possible patient loyalty to a physician working in another healthcare establishment.

Find hereunder a quick reminder in order to facilitate the reading of these Tables:

- $\quad$ issue a) is related to a patient who is not in the appropriate care unit,

- $\quad$ issue b) stands for the inadequacy between the room type that the patient initially wanted and what he received,

- $\quad$ issue c) is linked to a stay, longer than twenty-four hours, in the temporary emergency service,

- $\quad$ issue d) is related to the external transfers that are done.

Majority of problems, presented in Tables 1 and 2, can be differently considered according to the different decisional levels. All situations can be categorized as 
Table 1. Characteristics of hospitals and sum-up of the main encountered issues (mono site)

\begin{tabular}{|c|c|c|c|c|c|c|c|c|}
\hline \multirow[t]{2}{*}{ Hospital } & \multirow[t]{2}{*}{ Country } & \multirow[t]{2}{*}{ Size } & \multirow[t]{2}{*}{ Type } & \multirow[t]{2}{*}{ Mgt type } & \multicolumn{4}{|c|}{ Strategic/tactical/ operational } \\
\hline & & & & & a) & b) & c) & d) \\
\hline 1 & B & Big & $\mathrm{U}$ & S, GS & $\mathrm{X}$ & & & \\
\hline 2 & B & Big & G & S, GS & & $\mathrm{X}$ & $\mathrm{X}$ & \\
\hline 3 & B & Average & G & S, LOS & $\mathrm{X}$ & $\mathrm{X}$ & $\mathrm{X}$ & \\
\hline 4 & B & Average & G & S, GS, LOS & $\mathrm{X}$ & $\mathrm{X}$ & & \\
\hline 5 & B & Small & $\mathrm{U}$ & S & $\mathrm{X}$ & & & $\mathrm{X}$ \\
\hline 6 & B & Average & $\mathrm{U}$ & GS, LOS & $\mathrm{X}$ & $\mathrm{X}$ & & \\
\hline 7 & $\mathrm{Fr}$ & Average & $\mathrm{CH}$ & S, GS, LOS & & $\mathrm{X}$ & & $\mathrm{X}$ \\
\hline 8 & $\mathrm{Fr}$ & Average & $\mathrm{CH}$ & $\mathrm{S}, \mathrm{GS}$ & & & & \\
\hline 9 & Fr & Big & $\mathrm{CHU}$ & LOS & $\mathrm{X}$ & $\mathrm{X}$ & $\mathrm{X}$ & \\
\hline \multicolumn{5}{|c|}{ Number of occurrences } & 6 & 6 & 3 & 2 \\
\hline
\end{tabular}

Table 2. Characteristics of hospitals and sum-up of the main encountered issues (multi-site)

\begin{tabular}{|c|c|c|c|c|c|c|c|c|}
\hline \multirow[t]{2}{*}{ Hospital } & \multirow[t]{2}{*}{ Country } & \multirow[t]{2}{*}{ Size } & \multirow[t]{2}{*}{ Type } & \multirow[t]{2}{*}{ Mgt type } & \multicolumn{4}{|c|}{ Strategic/tactical/ operational } \\
\hline & & & & & a) & b) & c) & d) \\
\hline 10 & B & Big & GCU & S, GS & $\mathrm{X}$ & & $\mathrm{X}$ & \\
\hline 11 & B & Big & G & S, GS, LOS & & $\mathrm{X}$ & $\mathrm{X}$ & \\
\hline 12 & B & Big & G & S, GS, LOS & $\mathrm{X}$ & & $\mathrm{X}$ & \\
\hline 13 & B & Big & $\mathrm{U}$ & S, GS & $\mathrm{X}$ & & $\mathrm{X}$ & \\
\hline 14 & B & Average & G & GS, LOS & $\mathrm{X}$ & & & \\
\hline 15 & B & Average & G & LOS & $\mathrm{X}$ & $\mathrm{X}$ & & \\
\hline 16 & B & Average & G & $\mathrm{S}, \mathrm{GS}$ & $\mathrm{X}$ & & $\mathrm{X}$ & $\mathrm{X}$ \\
\hline 17 & B & Big & G & GS & $\mathrm{X}$ & $\mathrm{X}$ & & \\
\hline 18 & $\mathrm{Fr}$ & Big & $\mathrm{CHU}$ & GS, LOS & $\mathrm{X}$ & $\mathrm{X}$ & & \\
\hline \multicolumn{5}{|c|}{ Number of occurrences } & 8 & 4 & 5 & 1 \\
\hline
\end{tabular}

belonging to operational problems. Nevertheless, as these issues appear frequently, it is also possible to study them in the long-term i.e. to a strategic or tactical level. Additionally, hospitals were asked to precise which networks were existing inside their structures. Consequently, we mainly observed four main collaborations along the eighteen visited hospitals: multi-site structure, collaboration between two hospitals from different networks, collaboration with other structures and collaboration between two cross-border hospitals.

Some healthcare establishments are organized in a multi-site architecture. This permits a joint management and harmonizes the patients' flows when needed. Indeed, when the occupation rate is pretty high, patients can be welcomed in another site thanks to an internal transfer between both establishments. This naturally implies a surplus of work in terms of administration and stretcher-bearing activities. However, this has an impact on the care accessibilities when they are only provided on one site, especially regarding care units with a huge activity e.g. paediatrics, maternity service, emergency service and cardiology. 
As part of collaborations between two hospitals from different networks, some agreements are set up in order to ensure the continued good management in terms of financing and medical activity. It is also the case when one establishment cannot offer a specific treatment to patients e.g. cardiac coronagraph. The hospital sends thus its patients to another structure able to do the radiological exam. Collaborative networks between the hospital and other structures also exist. Some revalidation centres or rest homes sometimes have specific collaborations with hospitals.

Let us also note that collaboration between healthcare establishments and GP's from the area is sometimes set up. Indeed, some hospital software's allow general practitioners to have a look at patients' data, radiotherapy reports, etc. in order to better understand patients' medical history.

Collaborations between two countries also have an important impact on hospitals located near the border. As the financing is based on the activity rate, Belgium and France are desirous to welcome international patients.

\section{Reformulation of the Problem}

All problems explained in the previous section need to be reformulated. Indeed, all of them can be linked to an objective function with different aims: to maximize the profit in terms activity volume and if we welcome more patients, to maximize patients' satisfaction and finally to minimize the logistic costs and the waste of time.

As all encountered issues can be easily linked to the Figure 1, the objective function that will be calculated, will permit taking into account all the statements of facts. It will also be interesting to calculate this objective function and conclude if and how these problems can be resolved thanks to the creation of networks and collaborations between hospitals. Based on that, few examples of strategies can be foreseen and will be further explored: if there is no available bed, what is the best solution: to transfer in another hospital or cancel a planned hospitalization while it can

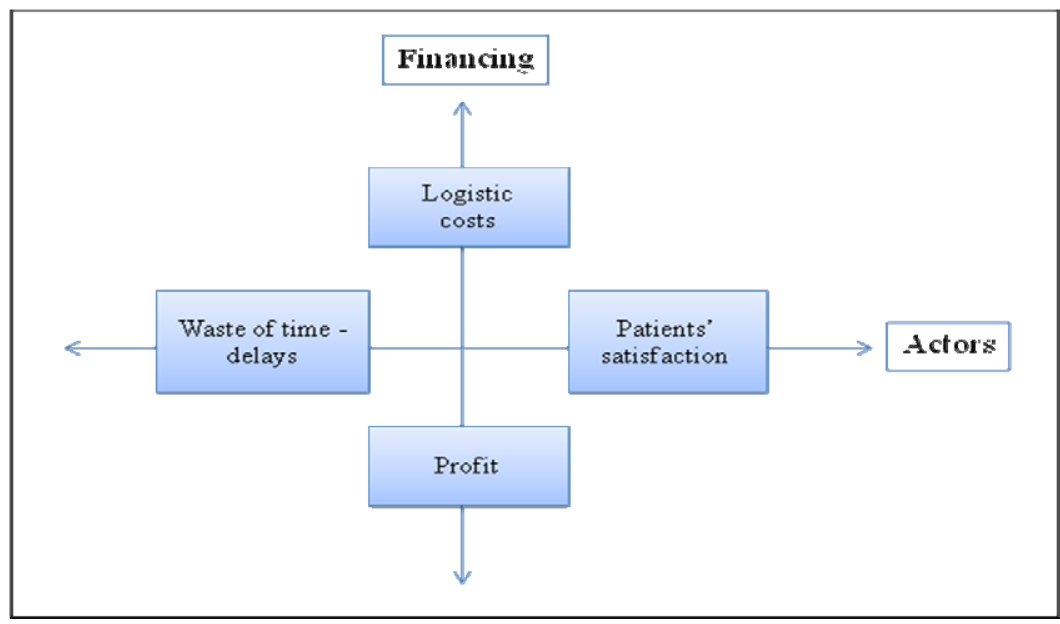

Fig. 1. Reformulation of the different issues 
damage the qualitative brand image of the hospital and cause an additional stress for the patient? In the same context, what would be the impact of the creation of a multipathologies temporary service if this temporary department is between the emergency room and the care units? In the same way, what would be the financial impact if all beds from different hospitals were grouped together and would thus accept patients from another site but directly in the appropriate care unit? The creation of networks between healthcare establishments lets us think about the interest of creating more collaborations with rehabilitation centers and rest homes, and even about the opportunity of studying the return-on-investment in case of building an additional unit dedicated to geriatrics or rehabilitation. It could be also interesting to study the impact of external, internal and intra-units transfers in terms of costs and patients' satisfaction, especially in the case of multi sites establishments. The impact of a duty period for different emergency rooms from different hospitals could also be interesting to study in the context of networks and especially in terms of financial impact and good patients' allocation in the appropriate care units.

However, started research will mainly concern the necessary of having a good vision in real time of the occupation rates, especially when the structure is laid out in several sites. The current idea is to further develop a patient's satisfaction survey, to cross findings with findings found thanks to the different visits and to analyze, in different angles, the added-value, strengths and weaknesses of these ideas.

\section{Conclusion and Perspectives}

Lots of elements and disruptions need to be taken into account when a bed planning is put in place, without forgetting the impact that it will have on the budget. Therefore, studying the encountered issues based on the structure type (mono site versus multisite) is crucial, especially regarding the strategic management and the routes of improvement that will be proposed in the future research.

Started research will thus work on one or few compromises evaluated on different criterions, quantitative (e.g. financial) or qualitative (patients' satisfaction). This satisfaction can be studied for example via a questionnaire oriented "managing/ planning of hospital beds" related to the whole hospitalization process while bringing to light the different stages of the patients' stay. It is therefore important to also get the patients' opinion about the main encountered issues related to hospital bed management. Within the framework of a multi-site structure, it could be interesting to compare the efficiency and costs of a bed manager for one or several structures versus the advantages linked to a subcontract, outside the healthcare establishments. Lots of constraints will also have an impact while optimizing the available resources: accepting the emergency case and therefore delaying the planned patient, adding additional beds within a care unit and studying at the same time the collateral costs, opening or closing beds related to a specialty, etc. Although this research is at its first stages, several avenues are still to be explored and courses of improving remain open. 


\section{References}

[1] Nguyen, M., Six, P., Antonioli, D., Glemain, P., Potel, G., Lombrail, P., Le Beux, P.: A simple method to optimize hospital beds capacity. International Journal of Medical Informatics 74(1), 39-49 (2005)

[2] Cochran, J.K., Roche, K.T.: A multi-class queuing network analysis methodology for improving hospital emergency department performance. Computers \& Operations Research 36(5), 1497-1512 (2009)

[3] Wang, T., Guinet, A., Besombes, B.: A sizing tool for allocation planning of hospital bed resources. In: Intelligent patient Management. SCI, vol. 189, pp. 113-125 (2009)

[4] Vassilacopoulos, G.: A simulation model for bed allocation to hospital inpatient departments. Simulation 45, 233-241 (1985)

[5] Walczak, S., Pofahl, W.E., Scorpio, R.J.: A decision support tool for allocating hospital bed resources and determining required acuity of care. Decision Support Systems 34, 445456 (2002)

[6] Ben Bachouch, R., Guinet, A., Hajrj-Gabouj, S.: Gestion des lits mutualisés d'un établissement hospitalier. In: 7e Congrès international de génie industriel, Trois-Rivières, Québec, Juin 5-8 (2007)

[7] Axelrod, R.: The evolution of cooperation. Basic books, New York (1984)

[8] Milward, H., Provan, K.: A manager's guide to choosing and using collaborative networks. IBM center for the business of governmment (2006) 